\title{
Complex bursting in pancreatic islets: a potential glycolytic mechanism
}

\author{
Keola Wierschem ${ }^{\mathrm{a}}$, Richard Bertram ${ }^{\mathrm{a}, \mathrm{b}, *}$ \\ ${ }^{a}$ Department of Mathematics, Florida State University, Tallahassee, FL 32306, USA \\ ${ }^{\mathrm{b}}$ Kasha Laboratory of Biophysics, Florida State University, Tallahassee, FL 32306, USA
}

Received 2 October 2003; received in revised form 28 January 2004; accepted 20 February 2004

\begin{abstract}
The electrical activity of insulin-secreting pancreatic islets of Langerhans is characterized by bursts of action potentials. Most often this bursting is periodic, but in some cases it is modulated by an underlying slower rhythm. We suggest that the modulatory rhythm for this complex bursting pattern is due to oscillations in glycolysis, while the bursting itself is generated by some other slow process. To demonstrate this hypothesis, we couple a minimal model of glycolytic oscillations to a minimal model for activitydependent bursting in islets. We show that the combined model can reproduce several complex bursting patterns from mouse islets published in the literature, and we illustrate how these complex oscillations are produced through the use of a fast/slow analysis. (C) 2004 Elsevier Ltd. All rights reserved.
\end{abstract}

Keywords: Insulin; Beta-cell; Bursting; Glycolysis

\section{Introduction}

$\beta$-cells are located in pancreatic islets of Langerhans and are responsible for the secretion of insulin following an elevation in the blood glucose level. The cells are electrically excitable, and $\mathrm{Ca}^{2+}$ brought in by action potentials evokes the secretion of insulin (Atwater et al., 1989). In in vitro recordings, $\beta$-cells display a bursting pattern of electrical impulses in the presence of stimulatory glucose concentrations $(>10 \mathrm{mM})$, consisting of an active phase of spiking followed by a silent phase of hyperpolarization (Dean and Mathews, 1970). The rate of insulin release is roughly proportional to the plateau fraction, i.e. the ratio of the active-phase duration to the total period (Atwater et al., 1989).

Measurements of $\beta$-cell electrical activity in mouse islets sometimes exhibit slow modulations of the bursting rhythm. We call this "complex bursting". In one study by Cook (1983) the normal 15-s (silent plus active phase) bursting was modulated by a slow variation in the plateau fraction. In this example of

*Corresponding author. Department of Mathematics, Florida State University, Tallahassee, FL 32306, USA. Tel.: + 1-850-644-7195; fax: + 1-850-644-4053.

E-mail address: bertram@math.fsu.edu (R. Bertram). complex bursting, which we call "accordion bursting", the plateau fraction exhibited an almost sinusoidal variation with a period of $5 \mathrm{~min}$ (see simulation in Fig. 7). In a study by Henquin and collaborators (Henquin et al., 1982), three examples of complex bursting were shown. In one example, the plateau fraction first increases, then decreases, and is followed by a "desert", during which the cell is hyperpolarized for several minutes. The duration of the longest spiking phase is several times longer than that of the shortest active phase, and indeed the cell appears to be transiently locked in a continuous spiking state (see simulation in Fig. 9). The second example is similar to the first, except that there are no deserts (see simulation in Fig. 11).

Finally, the third example consists of one short burst, followed by a long burst, and then a desert (see simulation in Fig. 12).

In these experimental recordings of mouse islet behavior, two oscillatory modes appear to co-exist: one for bursting, and a slower one that modulates the bursting. In this report, we describe one possible mechanism for these complex bursting patterns, with a key role played by oscillations in glycolysis.

Glycolysis is the first stage of aerobic cellular respiration. It converts glucose to products that are further processed in the mitochondria to produce 
adenosine triphosphate (ATP) from adenosine diphosphate (ADP). Oscillations in glycolysis have been observed in yeast (Betz and Chance, 1965; Danø et al., 1999), muscle extracts (Tornheim and Lowenstein, 1974, 1975), and $\beta$-cells (Longo et al., 1991). These oscillations are thought to be due to the allosteric enzyme phosphofructokinase (PFK), which phosphorylates fructose-6-phosphate (F6P) to form fructose 1,6-bisphosphate (FBP). The PFK activity is subject to feedback regulation by a number of factors, including positive feedback by its product FBP, positive ADP feedback, and negative ATP feedback (Goldbeter and Lefever, 1972; Tornheim and Lowenstein, 1975).

Our goals in this study were to determine whether the complex bursting patterns observed in $\beta$-cells can be explained by the interaction of a regular bursting rhythm with slower glycolytic oscillations, and to analyse the dynamics of this combined system. Several mathematical models have been developed for glycolytic oscillations. These differ in complexity and biological detail, but all focus on the action of PFK (Goldbeter and Lefever, 1972; Sel'kov, 1968; Smolen, 1995; Tornheim, 1979; Westermark and Lansner, 2003). We have chosen to use the model developed by Goldbeter and Lefever (1972) since this is sufficient to generate the required slow nucleotide oscillations and is sufficiently simple to allow for an analysis of the combined model. A more recent model by Westermark and Lansner (2003) gives a more accurate representation of the mechanism for glycolytic oscillations in $\beta$-cells, but it is more complex than the Goldbeter-Lefever model and it does not include a variable for the ATP concentration, which is needed here. The model used for the $\beta$-cell electrical activity is a variant of the Chay-Keizer model (Chay and Keizer, 1983), the first model developed for bursting in islets. Again, more accurate models now exist for islet bursting, but this simpler description is more convenient for the analysis performed in this study.

Pancreatic $\beta$-cells contain a number of ion channel types. Crucial for the glycolytic modulation is the ATPsensitive $\mathrm{K}^{+}$channel, which is activated by ADP and inhibited by ATP. When glucose levels are low, so too is the intracellular ATP concentration relative to ADP. In this case, most of the K(ATP) channels are open, yielding a large current $I_{\mathrm{K} \text { (ATP) }}$ that hyperpolarizes the cell. At stimulatory glucose levels the ATP concentration is large and most of the K(ATP) channels are closed (Cook et al., 1988), allowing the cell to burst. However, if glycolytic oscillations occur, then at a constant stimulatory glucose level the ATP concentration oscillates, producing oscillations in the $\mathrm{K}(\mathrm{ATP})$ current conductance. In this way, oscillations in glycolysis are reflected in the electrical activity of the cell.

We begin by describing the models for glycolytic oscillations and electrical bursting. We then demon- strate how the interaction of the two oscillators can reproduce the complex bursting patterns observed experimentally, and investigate the dynamics of the combined model using a fast/slow analysis.

\section{The mathematical models}

\subsection{Model for glycolytic oscillations}

The Goldbeter-Lefever model (Goldbeter and Lefever, 1972) describes oscillations that can occur in the ADP and ATP concentrations during glycolysis in yeast, where there is positive feedback of ADP onto the allosteric enzyme PFK (Fig. 1). While the feedback onto PFK is different in mammals (positive feedback of FBP rather than ADP, and negative feedback of ATP), in both yeast and mammals the end result is a slow oscillation in the nucleotide concentrations. The Goldbeter-Lefever model consists of two differential equations

$\frac{\mathrm{dATP}}{\mathrm{dt}}=(v-\Phi(\mathrm{ADP}, \mathrm{ATP})) / \tau_{c}$,

$\frac{\mathrm{dADP}}{\mathrm{dt}}=(\Phi(\mathrm{ADP}, \mathrm{ATP})-\eta \mathrm{ADP}) / \tau_{c}$,

where the function $\Phi$ is defined by

$\Phi(\mathrm{ADP}, \mathrm{ATP})=\mathrm{ATP}(1+k \mathrm{ADP})^{2}$.

For simplicity, we let ATP and ADP denote the nucleotide concentrations. The parameter $k=20$ is the ratio of the forward and reverse reaction rates of the substrate ATP binding with PFK to produce ADP. The time constant for the PFK reaction is represented by $\tau_{c} . \eta$ represents the degradation rate of ADP, while $v$ represents a constant influx of ATP into the system. The function $\Phi$ represents the PFK-catalyzed reaction. ATP is included in this function as an enzyme substrate and ADP is included as an allosteric activator.

The dynamics of the model can be understood through a phase plane analysis. The ATP and ADP nullclines are defined as curves in which $\mathrm{dATP} / \mathrm{d} t=0$

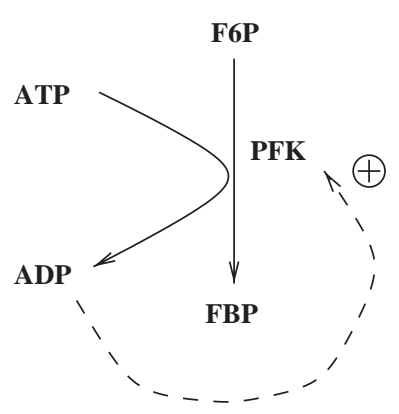

Fig. 1. Diagram of the action of PFK and positive feedback of ADP that is included in the Goldbeter-Lefever model. F6P and ATP are converted to FBP and ADP by PFK. 

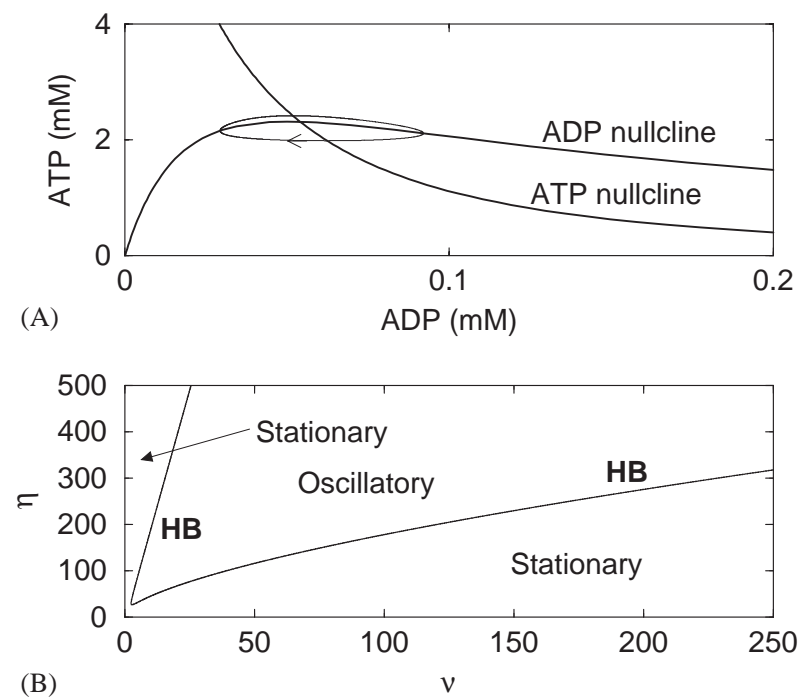

Fig. 2. (A) ADP and ATP nullclines with superimposed oscillatory trajectory $\left(\eta=185, v=10, \tau_{c}=1200 \mathrm{~s}\right)$. (B) Two-parameter diagram showing the regions in which glycolysis is stationary or oscillatory. The curves separating these regions are composed of HB points. $v$ and $\eta$ are dimensionless parameters. All simulations and bifurcation diagrams were generated with the XPPAUT software package (Ermentrout, 2002).

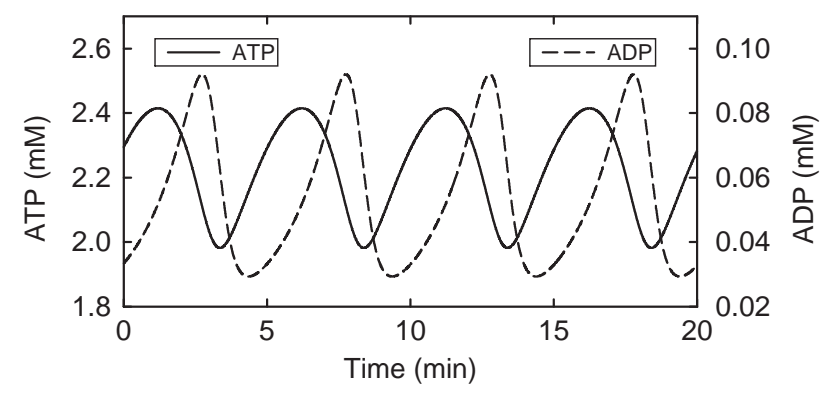

Fig. 3. Oscillations in the concentrations of ATP and ADP, with $\tau_{c}=$ $1200 \mathrm{~s}, \eta=185, v=10$.

and $\mathrm{dADP} / \mathrm{d} t=0$, respectively. The system equilibrium occurs at the intersection of the nullclines, and can be stable or unstable. When the equilibrium occurs at a location where the ADP nullcline has negative slope, as in Fig. 2A, the glycolytic system can oscillate. The period of the oscillation is set by the time constant $\tau_{c}$. Fig. 2B is a two-parameter bifurcation diagram showing the combinations of parameters $v$ and $\eta$ that yield oscillations. The curves separating the stationary and oscillatory regions of the $v \eta$-plane are composed of Hopf bifurcation points (HB). The large oscillatory region of the $v \eta$-plane suggests that oscillatory behavior is a robust feature of the model.

Fig. 3 shows one example of ADP and ATP time courses during oscillatory glycolysis. As ADP rises, PFK activity increases, converting more ATP to ADP and accelerating the rise of ADP. This accelerating PFK activity leads to a rapid decline in substrate ATP concentration, which decreases the enzyme activity and restarts the cycle.

\subsection{Model for electrical bursting}

Several models have been developed for bursting in pancreatic $\beta$-cells (Bertram et al., 2000; Chay, 1996; Chay and Keizer, 1983; Goforth et al., 2002; Keizer and Smolen, 1991; Sherman et al., 1988). These models differ primarily in the slow process responsible for driving the bursting. The first $\beta$-cell model was developed by Chay and Keizer (1983), based on the hypothesis of Atwater and Rojas (Atwater et al., 1980) that slow activitydependent oscillations in the cytosolic $\mathrm{Ca}^{2+}$ concentration drive the bursting via activation of a $\mathrm{Ca}^{2+}$-activated $\mathrm{K}^{+}$current. Although there is now evidence for additional slow processes (Bertram and Sherman, 2000), this model stands as a template for some of the more detailed models, and has the advantage of being relatively simple. We therefore employ a model based on that of Chay and Keizer, consisting of equations for voltage $(V), \mathrm{K}^{+}$channel activation $(n)$, and the free cytosolic $\mathrm{Ca}^{2+}$ concentration (c)

$\frac{\mathrm{d} V}{\mathrm{~d} t}=-\left[I_{\mathrm{Ca}}+I_{\mathrm{K}}+I_{\mathrm{K}(\mathrm{Ca})}+I_{\mathrm{K}(\mathrm{ATP})}\right] / C_{m}$,

$\frac{\mathrm{d} n}{\mathrm{~d} t}=\left[n_{\infty}(V)-n\right] / \tau_{n}$,

$\frac{\mathrm{d} c}{\mathrm{~d} t}=J_{\text {mem }}$.

The change in voltage is determined by the dynamics of the ionic currents, which include a $\mathrm{Ca}^{2+}$ current $\left(I_{\mathrm{Ca}}\right)$, a $\mathrm{K}^{+}$current $\left(I_{\mathrm{K}}\right)$, a Ca ${ }^{2+}$-activated $\mathrm{K}^{+}$current $\left(I_{\mathrm{K}(\mathrm{Ca})}\right)$, and an ATP-sensitive $\mathrm{K}^{+}$current $\left(I_{\mathrm{K}(\mathrm{ATP})}\right)$. These are defined as

$I_{\mathrm{Ca}}=\bar{g}_{\mathrm{Ca}} m_{\infty}(V)\left(V-V_{\mathrm{Ca}}\right)$,

$I_{\mathrm{K}}=\bar{g}_{\mathrm{K}} n\left(V-V_{\mathrm{K}}\right)$,

$I_{\mathrm{K}(\mathrm{Ca})}=\bar{g}_{\mathrm{K}(\mathrm{Ca})} \omega(c)\left(V-V_{\mathrm{K}}\right)$,

$I_{\mathrm{K}(\mathrm{ATP})}=\frac{\bar{g}_{\mathrm{K}(\mathrm{ATP})}}{\mathrm{ATP}}\left(V-V_{\mathrm{K}}\right)$,

where the steady-state functions are given by the increasing hyperbolic expressions

$m_{\infty}(V)=\left[1+\mathrm{e}^{\left(v_{m}-V\right) / s_{m}}\right]^{-1}$,

$n_{\infty}(V)=\left[1+\mathrm{e}^{\left(v_{n}-V\right) / s_{n}}\right]^{-1}$.

Activation of $I_{\mathrm{Ca}}$ is rapid, so for simplicity it is assumed to be instantaneous (Eq. (7)). The activation variable for $I_{\mathrm{K}(\mathrm{Ca})}, \omega$, is $\mathrm{Ca}^{2+}$ dependent and described by a hyperbolic function

$\omega(c)=\frac{c}{c+k_{D}}$. 
The form of the K(ATP) conductance, $\bar{g}_{\mathrm{K}(\mathrm{ATP})} / \mathrm{ATP}$, reflects the inverse dependence on the ATP concentration. Here we assume that ATP is non-zero (a valid assumption physiologically), and we use a non-saturating function for the ATP dependence since, in our simulations, the ATP concentration never approaches the extreme values where saturation would occur. It is through the K(ATP) conductance that glycolytic oscillations affect the membrane potential. When uncoupled from the glycolytic model the K(ATP) conductance is constant.

Finally, $\mathrm{Ca}^{2+}$ influx through the membrane is described by

$J_{\text {mem }}=-f\left(\alpha I_{\mathrm{Ca}}+k_{c} c\right)$,

where $f$ is the ratio of free to total $\mathrm{Ca}^{2+}, \alpha$ converts current to flux, and $k_{c} c$ represents pumping of $\mathrm{Ca}^{2+}$ out of the cell through plasma membrane $\mathrm{Ca}^{2+}$ ATPases. Parameter values are given in Table 1 , except for $\bar{g}_{\mathrm{K} \text { (ATP) }}$ and $f$ which are varied to yield different bursting rhythms. Values for these parameters are given in the figure captions.

Fig. 4A shows a typical bursting rhythm for the membrane model uncoupled from the glycolytic oscilla-

Table 1

Parameter values for the bursting model

\begin{tabular}{llll}
\hline Parameter & Value & Parameter & Value \\
\hline$g_{\mathrm{Ca}}$ & $1200 \mathrm{pS}$ & $g_{\mathrm{K}}$ & $3000 \mathrm{pS}$ \\
$g_{\mathrm{K}(\mathrm{Ca})}$ & $300 \mathrm{pS}$ & $\tau_{n}$ & $16 \mathrm{~ms}$ \\
$V_{\mathrm{Ca}}$ & $25 \mathrm{mV}$ & $V_{\mathrm{K}}$ & $-75 \mathrm{mV}$ \\
$C_{m}$ & $5300 \mathrm{fF}$ & $k_{c}$ & 0.1 \\
$k_{D}$ & $0.3 \mu \mathrm{M}$ & $\alpha$ & $2.25 \times 10^{-6} \mathrm{fA}^{-1} \mu \mathrm{M} \mathrm{ms}^{-1}$ \\
$s_{m}$ & $12 \mathrm{mV}$ & $v_{m}$ & $-20 \mathrm{mV}$ \\
$s_{n}$ & $5.6 \mathrm{mV}$ & $v_{n}$ & $-16 \mathrm{mV}$ \\
\hline
\end{tabular}
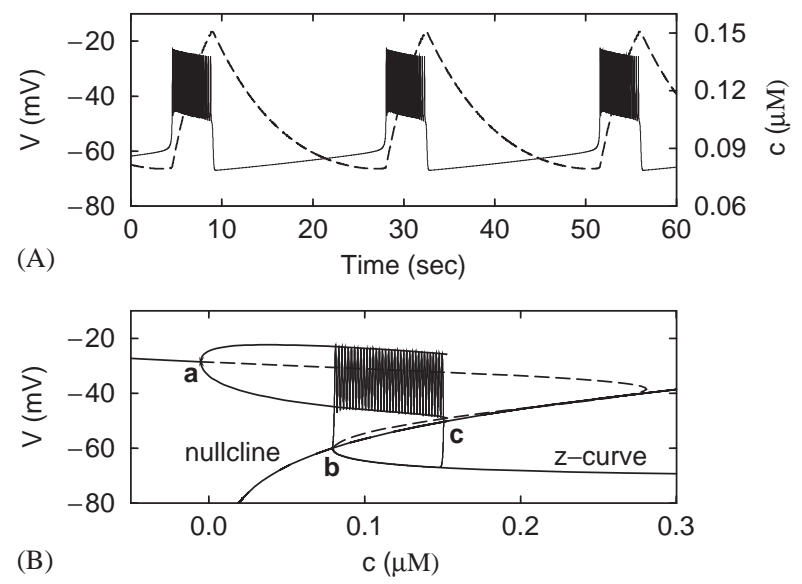

Fig. 4. (A) Bursting rhythm generated by the membrane model $\left(\bar{g}_{\mathrm{K} \text { (ATP) }}=170 \mathrm{pS}, f=0.001\right)$. Activity-dependent oscillations in the free cytosolic $\mathrm{Ca}^{2+}$ concentration (dashed) drive the bursting. (B) Fast/ slow analysis of bursting. The burst trajectory (oriented clockwise) is superimposed on the bifurcation diagram for the fast subsystem. The $c$ nullcline is also included. tor. When the $\mathrm{Ca}^{2+}$-concentration drops below a threshold value the magnitude of the hyperpolarizing $I_{\mathrm{K}(\mathrm{Ca})}$ is sufficiently small to put the cell into an active spiking phase. Each spike brings in $\mathrm{Ca}^{2+}$, which accumulates and activates more $I_{\mathrm{K}(\mathrm{Ca})}$. When the $\mathrm{Ca}^{2+}$ concentration accumulates to a sufficiently high level, $I_{\mathrm{K}(\mathrm{Ca})}$ prevents the cell from reaching the spike threshold and a silent phase commences.

The dynamics of the bursting oscillation are best understood using a fast/slow analysis, where the fast and slow dynamics are formally separated (Rinzel, 1985). This approach is described in detail in Rinzel (1985, 1987) and Bertram et al. (1995). In the bursting model, $c$ evolves on a slower time scale than $V$ and $n$, so in the fast/slow analysis it is treated as a parameter of the fast $V-n$ subsystem. A fast subsystem bifurcation diagram is then constructed with $c$ as the bifurcation parameter. The stationary branch is $z$-shaped (referred to as a "z-curve") with a stable lower branch that becomes unstable at a saddle-node bifurcation, an unstable middle branch of saddle points, and an unstable upper branch surrounded by a periodic branch of stable limit cycles (Fig. 4B). The periodic branch, reflecting trains of electrical impulses, emanates from a Hopf bifurcation (HB, location a) and terminates at an infinite-period homoclinic bifurcation (location c). The stable stationary branch terminates at a saddle-node bifurcation (location b).

The next step in the analysis is the superposition of the $c$-nullcline

$c=-\frac{\alpha}{k_{c}} I_{\mathrm{Ca}}$.

The dynamics of the full three-dimensional system can now be understood using the $z$-curve and $c$-nullcline. Below the $c$-nullcline the trajectory moves to the left; above the nullcline it moves to the right. Because of the difference in time scales, motion is always along stable branches of the fast substyem, except for rapid transitions.

Finally, the bursting trajectory is projected onto the $V-n$ plane. During the silent phase the trajectory moves leftward along the stationary branch until its termination at the saddle-node bifurcation at $\mathbf{b}$. From here, the system enters the active phase and moves rightward along the periodic branch. When the homoclinic bifurcation is reached the trajectory returns to the stationary branch, restarting the cycle.

\subsection{The composite model}

In the composite model, ATP from the glycolytic subsystem feeds into the model for electrical activity through the ATP-sensitive potassium channel conductance (Eq. (10)). The influence of ATP can be understood in terms of the fast/slow analysis (Fig. 5). ATP has 


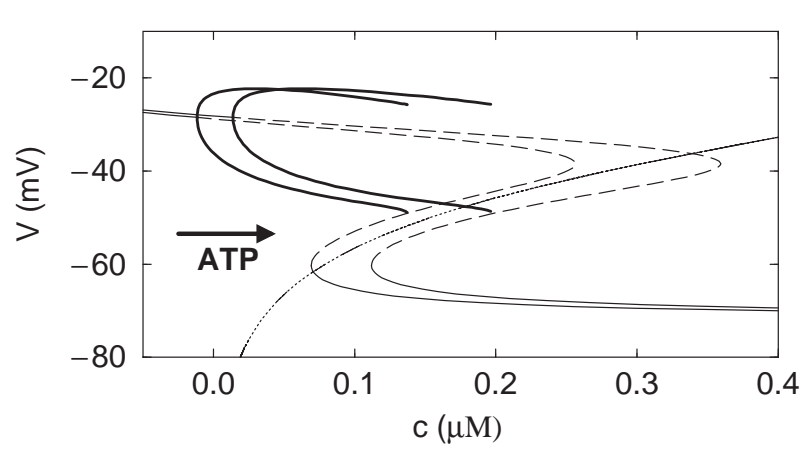

Fig. 5. Increasing ATP concentration moves the $z$-curve to the right.
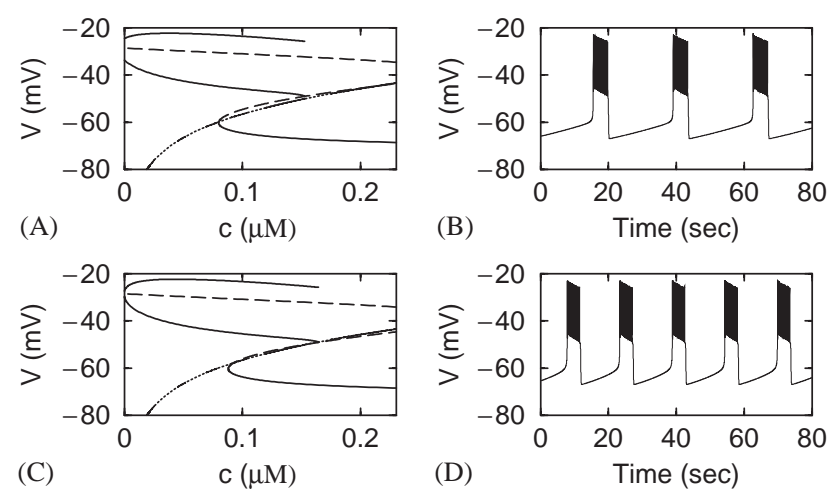

(B)

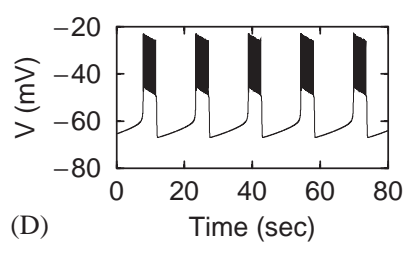

Fig. 6. Illustration of the effects of the nullcline location on the plateau fraction. (A,B) At low ATP values the $c$ nullcline crosses the $z$ curve close to the saddle node bifurcation, producing bursting with a low plateau fraction $\left(\bar{g}_{\mathrm{K}(\mathrm{ATP})}=170 \mathrm{pS}, f=0.001\right)$. (C,D) At higher ATP values the $c$ nullcline intersects the $z$-curve closer to the homoclinic bifurcation, reducing the silent-phase duration which results in bursting with a larger plateau fraction $\left(\bar{g}_{\mathrm{K}(\mathrm{ATP})}=165 \mathrm{pS}\right.$, $f=0.001)$.

no effect on the $c$ nullcline (Eq. (15)), and only effects the $c$ dynamics indirectly through $V$. However, it does affect voltage (Eqs. (4) and (10)), and thus influences the $z$-curve. In particular, increasing ATP moves the $z$-curve to the right. This is because the increase in ATP reduces the conductance of the K(ATP) current, so more $\mathrm{K}(\mathrm{Ca})$ current is needed to bring the cell to rest, and the $z$-curve is shifted to higher levels of $c$.

If ATP is low, then the nullcline intersects the bottom branch of the $z$-curve and the cell is at rest. At higher ATP levels the intersection occurs on the middle branch and the cell bursts. If the intersection is low on the middle branch, near the saddle-node bifurcation, then the bursting trajectory moves rapidly along the periodic branch and slowly along the stationary branch, resulting in bursting with a low plateau fraction (Fig. 6A,B). If the intersection occurs higher up on the middle branch, as would be the case with a higher ATP concentration (lower $g_{\mathrm{K}(\mathrm{ATP})}$ ), then the trajectory moves rapidly along the stationary branch and slowly along the periodic branch and the bursting that is produced has a high plateau fraction (Fig. 6C,D).

At very high ATP concentrations, the $z$-curve is translated so far to the right that the nullcline intersects the periodic branch resulting in continuous spiking.

\section{Complex bursting}

\subsection{Accordion bursting}

Our first use of the composite model is to simulate the "accordion bursting" observed by Cook (1983), which is characterized by a slow variation of the burst plateau fraction. This complex bursting oscillation can be understood in terms of the fast/slow analysis. When glycolytic oscillations occur, the variations in ATP move the $z$-curve back and forth, shifting the location at which the $c$ nullcline intersects the $z$-curve. While the intersection always occurs on the middle branch of the $z$-curve, it is sometimes closer to the saddle node and sometimes closer to the homoclinic bifurcation. As a result, the bursting rhythm smoothly alternates between a low(Fig. 6A,B) and a high plateau fraction (Fig. 6C,D). This is shown in Fig. 7. Panel A shows the simulated accordion bursting oscillation, while the bottom panels quantify some of the features of the oscillation. Panel B shows a profound rhythm in the plateau fraction, which ranges from roughly 0.3 to 0.6 with a period of $5 \mathrm{~min}$
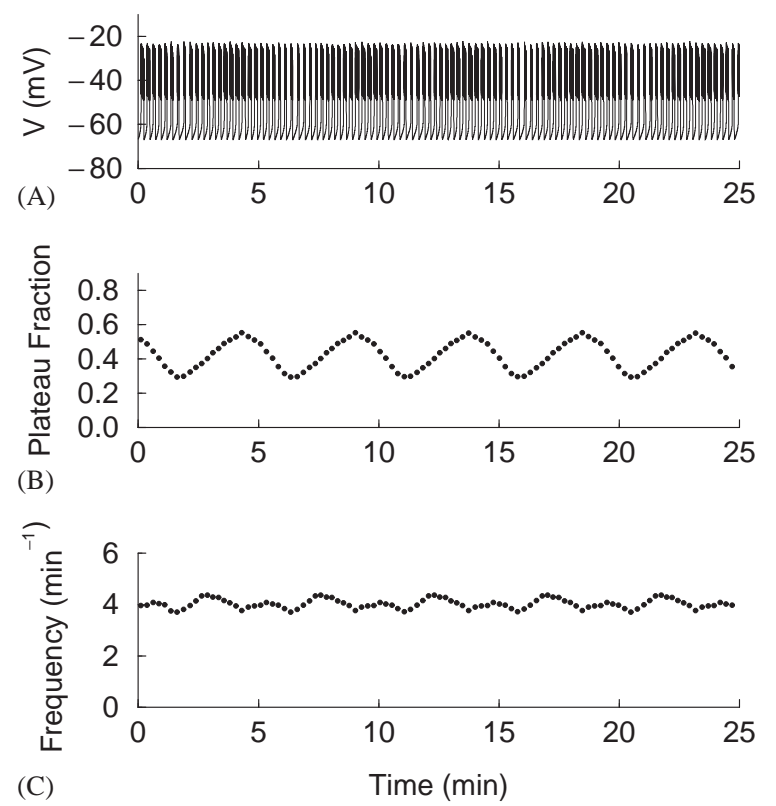

Fig. 7. Simulation of accordion bursting pattern $\left(\bar{g}_{\mathrm{K}(\mathrm{ATP})}=357 \mathrm{pS}\right.$, $f=0.0013, v=10, \eta=188, \tau_{c}=1200 \mathrm{~s}$ ). (A) Complex "accordion bursting" pattern with rhythmic plateau modulation. (B) Rhythmic variation in the plateau fraction. (C) Oscillations in burst frequency are twice as fast as oscillations in plateau fraction. Characteristics of this accordion bursting are similar to the bursting described in Fig. 1 of Cook (1983). 
(the period of the glycolytic oscillation). Panel $\mathrm{C}$ shows rhythmic variation in the burst frequency, which is twice as fast as the plateau fraction rhythm. These simulation data are similar to the experimental data from mouse islets shown by Cook (Fig. 1 of Cook, 1983).

The faster burst frequency rhythm in Fig. 7C, twice that of the glycolytic oscillation frequency, is due to the non-monotonic response of the burst frequency to an elevation in the slow nullcline (Carroll et al., 1990). When the nullcline is just above the saddle node, the plateau fraction and burst frequency are both low. As the nullcline is raised (or the $z$-curve is right-shifted by an increase in ATP), the plateau fraction increases monotonically. However, the burst frequency first increases due to a decrease in the silent-phase duration, but then decreases due to an increase in the active-phase duration. Thus, as ATP rises from trough to peak, the burst frequency first rises and then falls, yielding an oscillation with frequency twice that of the glycolytic frequency.

\subsection{Other examples of complex bursting}

We next apply the composite model to the three examples of complex bursting described by Henquin (Henquin et al., 1982). Again, these different oscillations can be decomposed using a fast/slow analysis. If the $z$-curve is shifted far to the left, the $c$ nullcline intersects the $z$-curve on the lower stable stationary branch. There is no bursting rhythm, and instead the system is at rest (Fig. 8A,B). If the $z$-curve is shifted far to the right, the $c$ nullcline intersection occurs on the periodic branch of the $z$-curve. The system then spikes continuously (Fig. 8C,D).

The complex bursting shown in Fig. 9 is similar to accordion bursting, in that ATP oscillates due to oscillations in glycolysis. However, the amplitude of
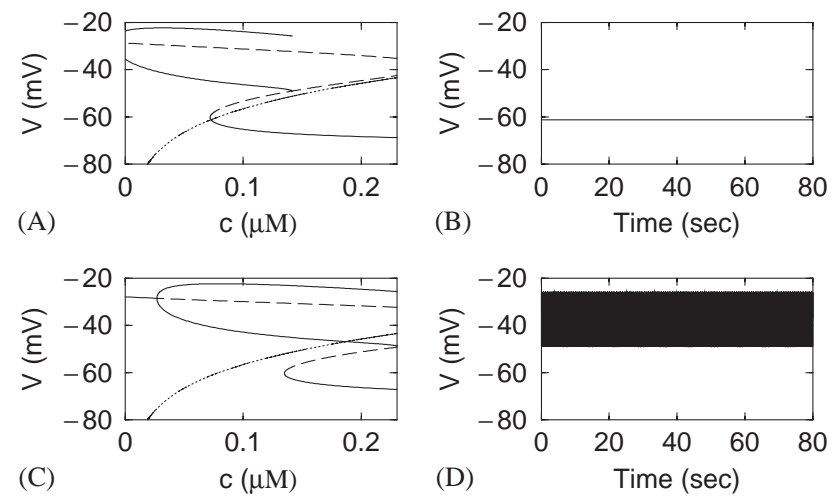

Fig. 8. (A,B) At sufficiently low values of ATP the $c$ nullcline intersects the $z$-curve on the bottom branch, and the system is at rest $\left(\bar{g}_{\mathrm{K}(\mathrm{ATP})}=175 \mathrm{pS}, f=0.001\right)$. (C,D) At sufficiently high values of ATP the nullcline intersects the periodic branch of the $z$-curve and the system spikes continuously $\left(\bar{g}_{\mathrm{K}(\mathrm{ATP})}=140 \mathrm{pS}, f=0.001\right)$.
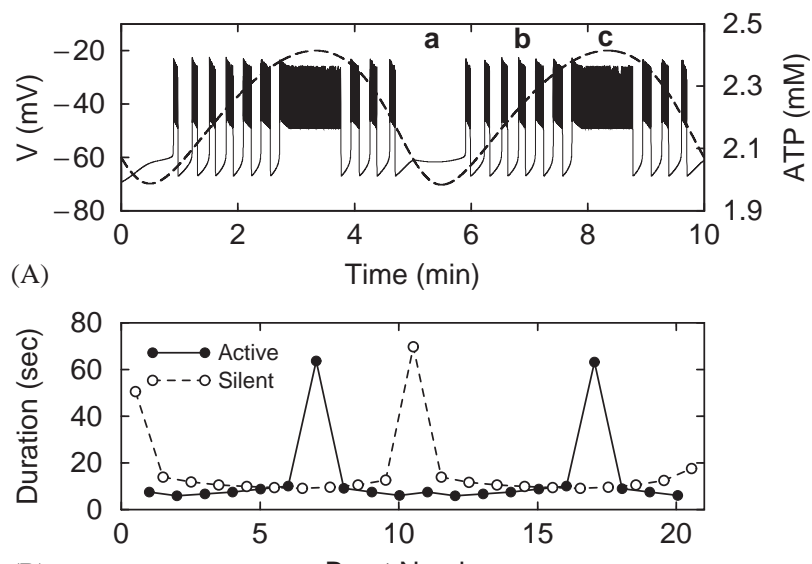

(B)

Burst Number

Fig. 9. (A) The bursting pattern exhibits a rhythmic plateau fraction, and periodic long spiking phases and deserts. The variation in bursting is due to a slow oscillation in ATP (dashed). The labels $\mathbf{a}, \mathbf{b}$, and $\mathbf{c}$ refer to the $z$-curves in Fig. 10. (B) Silent- and active-phase durations for each burst. Parameter values are $\bar{g}_{\mathrm{K}(\mathrm{ATP})}=350 \mathrm{pS}, f=0.001, v=10$, $\eta=185, \tau_{c}=1200 \mathrm{~s}$. This bursting is similar to the experimental recording from Henquin et al (1982), Fig. 2A.

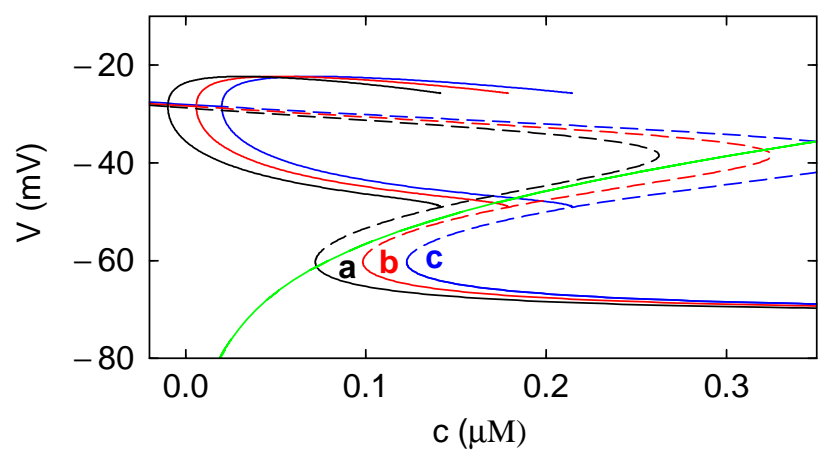

Fig. 10. The $c$-nullcline (green) and $z$-curves corresponding to the three phases of complex bursting labeled $\mathbf{a}, \mathbf{b}$, and $\mathbf{c}$ in Fig. 9. When ATP is low $g_{\mathrm{K} \text { (ATP) }}$ is large and the $z$-curve is left-shifted, putting the model cell into a rest state $(\mathbf{a}$, ATP $=2 \mathrm{mM})$. As ATP increases the $z$-curve moves to the right, producing transient bursting (b, ATP $=$ $2.2 \mathrm{mM}$ ), followed by a transient phase of continuous spiking $(c$, ATP $=2.5 \mathrm{mM})$. The cycle reverses during the downswing of the ATP oscillation.

the ATP oscillation is now greater. At the trough of the oscillation, the $z$-curve is shifted far to the left and the intersection with the nullcline is on the bottom branch (Fig. 10, black), so the voltage is repolarized (labeled a in Fig. 9). As ATP rises, the $z$-curve moves slowly rightward until the nullcline intersects the $z$-curve on the middle branch (Fig. 10, red), initiating several cycles of bursting driven by activity-dependent oscillations in $c$ (labeled b in Fig. 9). As the $z$-curve continues to move rightward, the intersection occurs higher up on the middle branch, resulting in an increasing plateau fraction. Eventually the $z$-curve moves so far to the right that the intersection occurs on the periodic branch (Fig. 10, blue), and the system enters a phase of 
continuous spiking (labeled $\mathbf{c}$ in Fig. 9). This spiking phase continues for as long as ATP is above the threshold value, which may be for a minute or longer. The long duration of the spiking phase, which is on the slow time scale of ATP oscillations, contrasts with the relatively short active phase of a burst, which is on the much faster time scale of $c$.

In the descending limb of the ATP oscillation the $z$-curve moves to the left, slowly progressing through the continuous spiking phase, then the bursting phase with ever shorter plateau fractions, and finally into an extended silent phase or "desert" where the nullcline intersects the bottom branch of the $z$-curve. The variations in active and silent phase durations are quantified in Fig. 9B. There is clearly a longer active phase duration when the phase point is stalled on the periodic branch, and a long silent phase duration when it is stalled on the lower stationary branch. This form of complex bursting, with modulated plateau fraction, periodic long spiking phases and deserts, is similar to experimental islet data in Fig. 2A of Henquin et al. (1982).

Another example of complex bursting, this time without deserts, is shown in Fig. 11. Again, there is a slow variation in the silent and active-phase durations (Fig. 11B) due to glycolytic oscillations in ATP. Although ATP rises to a level sufficiently high to produce a long spiking phase, it never declines to a level sufficiently low to produce a desert. This example of complex bursting is similar to experimental data in Fig. 2B of Henquin et al. (1982).

Fig. 12 illustrates complex bursting that appears to be quite different from the other examples, consisting of a desert, followed by a short burst, followed by a very long burst. This behavior is reflected in distinct rhythms
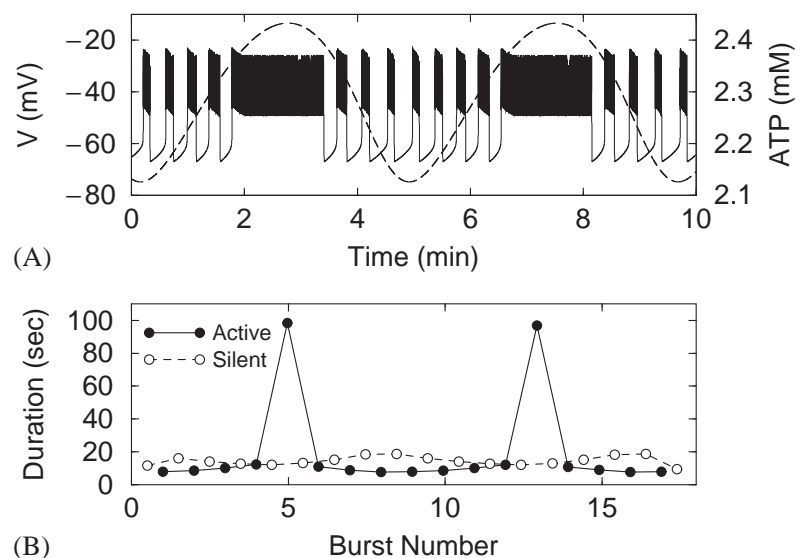

Fig. 11. (A) The bursting pattern exhibits a rhythmic plateau fraction highlighted with periodic long spiking phases, but with no deserts. (B) There are variations in active- and silent-phase durations, with periodic spikes in the active-phase duration when the trajectory is stalled on the periodic branch. Parameter values are $\bar{g}_{\mathrm{K}(\mathrm{ATP})}=350 \mathrm{pS}$, $f=0.00065, v=10, \eta=187.5, \tau_{c}=1200 \mathrm{~s}$. This bursting is similar to the experimental recording from Henquin et al. (1982), Fig. 2B.
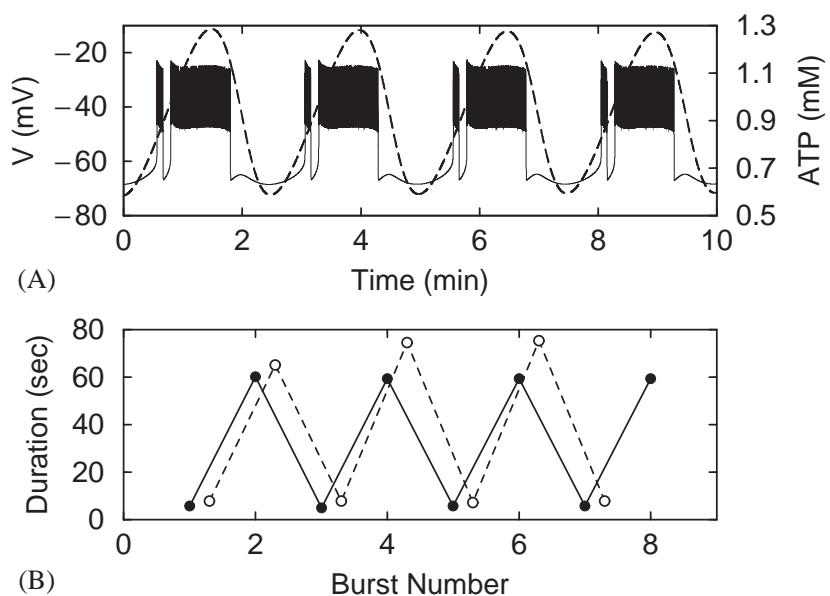

Fig. 12. (A) One "normal" burst is produced between each desert and long spiking phase. (B) There are regular oscillations between short and long active and silent phases in this example of complex bursting. Parameter values are $\bar{g}_{\mathrm{K}(\mathrm{ATP})}=150 \mathrm{pS}, f=0.0005, v=250, \eta=320$, $\tau_{c}=7000 \mathrm{~s}$. This bursting is similar to the experimental recording from Henquin et al. (1982), Fig. 2C.

in the active and silent-phase durations (Fig. 12B). As in other cases, the desert is due to a left-shifted $z$-curve, and the long spiking phase is due to a right-shifted curve. However, the region for which "normal" bursting is achieved, driven by an oscillation in $c$ as in Fig. 4, is small in this case. Thus, as ATP oscillates the system moves rapidly between a desert and a long burst, with only a single "normal" burst produced between. This is in contrast to earlier examples of complex bursting, where many cycles of bursting occur before and after a long spiking phase. This example of complex bursting is similar to experimental data in Fig. 2C of Henquin et al. (1982).

\section{Discussion}

We have demonstrated that the interaction of a bursting electrical oscillation with a slow glycolytic oscillation can produce complex electrical bursting patterns of the type published earlier from mouse pancreatic islets (Cook, 1983; Henquin et al., 1982). While this does not prove that the reported complex bursting patterns are produced through this mechanism, it does demonstrate the sufficiency of the glycolytic mechanism. We have also demonstrated how a complex bursting pattern can be analysed mathematically when one of the two slow processes is much slower than the other and is independent of electrical activity.

The role that glycolytic oscillations play in the electrical activity of islets is controversial. Some suggest that the regular bursting oscillations typically observed in islets are driven by glycolytic oscillations (Longo et al., 1991; Tornheim, 1997), while others suggest that nucleotide oscillations are driven by oscillations in 
$\mathrm{Ca}^{2+}$ that result from bursting (Ainscow and Rutter, 2002; Detimary et al., 1998; Keizer and Magnus, 1989; Kennedy et al., 2002). We suggest a third scenario: glycolytic oscillations, when they occur, can modulate the bursting that is produced by some other mechanism. The electrical recordings from Cook (1983) and Henquin et al. (1982) are examples of this modulated bursting, as are more recent intracellular calcium recordings from Zhang et al. (2003).

If our hypothesis is correct, then if glycolytic oscillations are somehow stopped complex bursting should transform into regular periodic bursting. If, on the other hand, the bursting is driven by glycolytic oscillations, then once the oscillations are stopped the bursting should stop. Experimentally, glycolytic oscillations can be controlled by controlling the environment. In principle, then, it should be possible to stop glycolytic oscillations in $\beta$-cells. In practice, however, there are many technical difficulties and questions about interpretation. In one example where clear oscillations in a metabolic indicator (oxygen content) were observed, the indicator stopped oscillating when the cell was hyperpolarized by application of a K(ATP) channel activator (Kennedy et al., 2002). This suggests that the electrical bursting was driving metabolic oscillations, possibly through the effects of calcium on mitochondrial metabolism. However, since electrical activity was not measured, it is not clear whether the bursting was regular or complex. The results may be different if the same protocol were applied to an islet exhibiting complex bursting. If our hypothesis is true, that glycolytic oscillations typically lead to complex bursting, then another difficulty in studying them is that they likely occur only rarely. The majority of the published recordings of islet electrical activity show regular periodic bursts, not the complex patterns we attribute to glycolytic oscillations. The rarity of glycolytic oscillations make them difficult to study.

The models used in this study were chosen to be as simple as possible to facilitate the fast/slow analysis. The same dynamic mechanisms would apply to complex bursting produced with more complete models as long as the glycolysis is independent of electrical activity and is slower than the slow processes driving bursting. Some more complete models for glycolytic oscillations include those by Smolen (Smolen, 1995), Tornheim (1979), and Westermark and Lansner (2003). More recent models of bursting in $\beta$-cells include those by Chay (1996), Keizer and Smolen (1989), and Sherman et al. (1988). In one recent $\beta$-cell model, the Phantom Bursting Model (PBM), bursting is driven by the interaction of more than one slow variable (Bertram et al., 2000; Goforth et al., 2002). In the PBM, the burst period ranges from a few seconds to a few minutes, depending upon the conductance of the various ionic currents. The fastest bursting is driven by the faster of the two slow variables, while the slowest bursting is driven by the other slow variable. The "medium" bursting typically exhibited by islets is due to the interaction of both slow variables. In the model used in the current study, the burst period is most effectively varied through the parameter $f$. Reducing $f$ slows down the bursting by reducing the speed of the single slow variable, the cytosolic-free $\mathrm{Ca}^{2+}$ concentration. We have set this parameter, the fraction of free $\mathrm{Ca}^{2+}$, to small values to obtain the rather long burst periods exhibited by the data. (A more realistic value of this parameter is $f=0.01$.) This could have been done more naturally by adjusting appropriate conductance values in the PBM, but at the cost of extra dynamical complexity.

Another parameter varied to give the various complex bursting patterns was the maximal K(ATP) conductance,

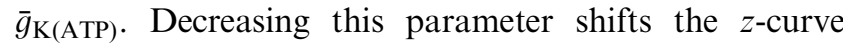
rightward, simulating the effects of increasing the glucose concentration. Finally, the parameters $v, \eta$, and $\tau_{c}$, which correspond to the glycolytic subsystem, were varied to change the speed of the glycolytic oscillation. Experimental data suggests that such oscillations have a wide range of periods, from a few minutes to more than ten minutes (Tornheim, 1997).

The major simplification made in this modeling study is the omission of metabolic processes downstream of glycolysis. The products of glycolysis are the substrates for the citric acid cycle, which in turn yields substrates for oxidative phosphorylation. Indeed, it is the latter process that produces most of the ATP. However, a model that includes these complex processes, even if in a simplified form, would make the fast/slow analysis performed here much more difficult and less transparent. In any case, oscillations in glycolysis should result in oscillations in the cytoplasmic level of ATP since the output of glycolysis is the input to downstream processes. Hence, we view the simulation results reported here as suggestive of what could occur if the full metabolic pathway were included in the model. Most importantly, the close similarity between the simulation results and experimental recordings suggest that some of the complex bursting patterns observed in islets may be due to an independent ATP oscillation, possibly of glycolytic origin.

\section{Acknowledgements}

This work was supported by National Science Foundation Grants DMS-9981822 and DMS-0311856 to R. Bertram.

\section{References}

Ainscow, E.K., Rutter, G.A., 2002. Glucose-stimulated oscillations in free cytosolic ATP concentration imaged in single islet $\beta$-cells. Diabetes 51, S162-S170. 
Atwater, I., Dawson, C.M., Scott, A., Eddlestone, G., Rojas, E., 1980. The nature of the oscillatory behavior in electrical activity for pancreatic $\beta$-cells. In: Thieme, G. (Ed.), Biochemistry and Biophysics of the Pancreatic $\beta$-cell. Verlag, New York, pp. 100-107.

Atwater, I., Carroll, P., Li, M.X., 1989. Electrophysiology of the pancreatic $\beta$-cell. In: Drazin, B., Melmed, S., LeRoith, D. (Eds.), Insulin Secretion. Alan R. Liss, Inc., New York, pp. 49-68.

Bertram, R., Sherman, A., 2000. Dynamical complexity and temporal plasticity in pancreatic $\beta$-cells. J. Biosci. 25, 197-209.

Bertram, R., Butte, M.J., Kiemel, T., Sherman, A., 1995. Topological and phenomenological classification of bursting oscillations. Bull. Math. Biol. 57, 413-439.

Bertram, R., Previte, J., Sherman, A., Kinard, T.A., Satin, L.S., 2000. The phantom burster model for pancreatic $\beta$-cells. Biophys. J. 79, 2880-2892.

Betz, A., Chance, B., 1965. Phase relationship of glycolytic intermediates in yeast cells with oscillatory metabolic control. Arch. Biochem. Biophys. 109, 585-594.

Carroll, P.B., Sherman, A., Furer, R., Baschero, A.C., Rinzel, J., Atwater, I., 1990. Modulation of the frequency of glucosedependent bursts of electrical activity by $\mathrm{HCO}_{3} / \mathrm{CO}_{2}$ in rodent pancreatic $\beta$-cells: experimental and theoretical results. Eur. Biophys. J. 18, 71-77.

Chay, T.R., 1996. Electrical bursting and luminal calcium oscillation in excitable cell models. Biol. Cybern. 75, 419-431.

Chay, T.R., Keizer, J., 1983. Minimal model for membrane oscillations in the pancreatic $\beta$-cell. Biophys. J. 42, 181-190.

Cook, D.L., 1983. Isolated islets of Langerhans have slow oscillations of electrical activity. Metabolism 32, 681-685.

Cook, D.L., Satin, L.S., Ashford, M.J., Hales, C.N., 1988. ATPsensitive $\mathrm{K}^{+}$channels in pancreatic $\beta$-cells: spare-channel hypothesis. Diabetes 37, 495-498.

Danø, S., Sørensen, P.G., Hynne, F., 1999. Sustained oscillations in living cells. Nature 402, 320-322.

Dean, P.M., Mathews, E.K., 1970. Glucose-induced electrical activity in pancreatic islet cells. J. Physiol. (London) 210, 255-264.

Detimary, P., Gilon, P., Henquin, J.-C., 1998. Interplay between cytoplasmic $\mathrm{Ca}^{2+}$ and the ATP/ADP ratio: a feedback control mechanism in mouse pancreatic islets. Biochem. J. 333, 269-274.

Ermentrout, G.B., 2002. Simulating, Analyzing, and Animating Dynamical Systems: A Guide to XPPAUT for Researchers and Students. SIAM Books, Philadelphia.

Goforth, P.B., Bertram, R., Khan, F.A., Zhang, M., Sherman, A., Satin, L.S., 2002. Calcium-activated $\mathrm{K}^{+}$channels of mouse $\beta$-cells are controlled by both store and cytoplasmic $\mathrm{Ca}^{2+}$ : experimental and theoretical studies. J. Gen. Physiol. 120, 307-322.
Goldbeter, A., Lefever, R., 1972. Dissipative structures for an allosteric model; application to glycolytic oscillations. Biophys. J. 12, 1302-1315.

Henquin, J.C., Meissner, H., Schmeer, W., 1982. Cyclic variations of glucose-induced electrical activity in pancreatic $\beta$-cells. Eur. J. Physiol. 393, 322-327.

Keizer, J., Magnus, G., 1989. ATP-sensitive potassium channel and bursting in the pancreatic $\beta$ cell. Biophys. J. 56, 229-242.

Keizer, J., Smolen, P., 1991. Bursting electrical activity in pancreatic $\beta$ cells caused by $\mathrm{Ca}^{2+}$ - and voltage-inactivated $\mathrm{Ca}^{2+}$ channels. Proc. Natl Acad. Sci. USA 88, 3897-3901.

Kennedy, R.T., Kauri, L.M., Dahlgren, G.M., Jung, S.-K., 2002. Metabolic oscillations in $\beta$-cells. Diabetes 51, S152-S161.

Longo, E.A., Tornheim, K., Deeney, J.T., Varnum, B.A., Tillotson, D., Prentki, M., Corkey, B.E., 1991. Oscillations in cytosolic free $\mathrm{Ca}^{2+}$, oxygen consumption, and insulin secretion in glucosestimulated rat pancreatic islets. J. Biol. Chem. 266, 9314-9319.

Rinzel, J., 1985. Bursting oscillations in an excitable membrane model. In: Sleeman, B.D., Jarvis, R.J. (Eds.),, Ordinary and Partial Differential Equations, Vol. 1151. Springer, Berlin, pp. 304-316.

Rinzel, J., 1987. A formal classification of bursting mechanisms in excitable systems. In: Teramoto, E., Yamaguti, M. (Eds.), Mathematical Topics in Population Biology, Morphogenesis and Neurosciences, Lecture Notes in Biomathematics, Vol. 71. Springer, Berlin, pp. 267-281.

Sel'kov, E.E., 1968. Self-oscillations in glycolysis. Eur. J. Biochem. 4, $79-86$.

Sherman, A., Rinzel, J., Keizer, J., 1988. Emergence of organized bursting in clusters of pancreatic $\beta$-cells by channel sharing. Biophys. J. 54, 411-425.

Smolen, P., 1995. A model for glycolytic oscillations based on skeletal muscle phosphofructokinase kinetics. J. Theor. Biol. 174, 137-148.

Tornheim, K., 1979. Oscillations of the glycolytic pathway and the purine nucleotide cycle. J. Theor. Biol. 79, 491-541.

Tornheim, K., 1997. Are metabolic oscillations responsible for normal oscillatory insulin secretion? Diabetes 46, 1375-1381.

Tornheim, K., Lowenstein, J.M., 1974. The purine nucleotide cycle. IV. Interactions with oscillations of the glycolytic pathway in muscle extracts. J. Biol. Chem. 249, 3241-3247.

Tornheim, K., Lowenstein, J.M., 1975. The purine nucleotide cycle. Control of phosphofructokinase and glycolytic oscillations in muscle extracts. J. Biol. Chem. 250, 6304-6314.

Westermark, P.O., Lansner, A., 2003. A model of phosphofructokinase and glycolytic oscillations in the pancreatic $\beta$-cell. Biophys. J. 85, 126-139.

Zhang, M., Goforth, P., Bertram, R., Sherman, A., Satin, L., 2003. The $\mathrm{Ca}^{2+}$ dynamics of isolated mouse $\beta$-cells and islets: implications for mathematical models. Biophys. J. 84, 2852-2870. 